Revue internationale P.M.E.

Économie et gestion de la petite et moyenne entreprise

\title{
Organisation industrielle, degré d'intégration bancaire des PME et analyse du risque
}

\section{Christian Picory}

Volume 8, numéro 3-4, 1995

URI : https://id.erudit.org/iderudit/1008360ar

DOI : https://doi.org/10.7202/1008360ar

Aller au sommaire du numéro

Éditeur(s)

Presses de l’Université du Québec

ISSN

0776-5436 (imprimé)

1918-9699 (numérique)

Découvrir la revue

Citer cet article

Picory, C. (1995). Organisation industrielle, degré d'intégration bancaire des PME et analyse du risque. Revue internationale P.M.E., 8(3-4), 91-120.

https://doi.org/10.7202/1008360ar
Résumé de l'article

Alors que le financement bancaire demeure la principale modalité de financement de la dynamique des PME, de récentes études ont montré qu'en matière d'accès aux concours bancaires, ces firmes sont pénalisées en raison, d'une part, de leur taille et, d'autre part, de procédures d'évaluation du risque qui leurs seraient défavorables. L’objet de cet article est précisément d'examiner l'idée même de «risque PME» en tenant compte, premièrement, du degré d'intégration bancaire de l'activité de ces entreprises et, deuxièmement, de l'organisation industrielle dans laquelle elles s'inscrivent. Après avoir précisé les concepts de «risque de financement» et de "risque industriel», l'analyse montrera que le critère d'organisation industrielle constitue un critère pertinent de différenciation du risque de financement et du degré d'intégration bancaire de l'activité productive qui lui est associé. L'appréciation du risque de financement selon le critère de la taille apparaît dès lors particulièrement contre-productive.
Ce document est protégé par la loi sur le droit d'auteur. L'utilisation des services d'Érudit (y compris la reproduction) est assujettie à sa politique d'utilisation que vous pouvez consulter en ligne.

https://apropos.erudit.org/fr/usagers/politique-dutilisation/ 


\title{
Organisation industrielle, degré d'intégration bancaire des PME et analyse du risque ${ }^{1}$
}

\author{
Christian PICORY* \\ École nationale supérieure des télécommunications
}

MOTS CLÉS

\author{
Organisation industrielle - Risque industriel \\ Risque de financement - Intégration bancaire de l'activité \\ productive - Relation Banque-PME
}

\begin{abstract}
RÉSUMÉ
Alors que le financement bancaire demeure la principale modalité de financement de la dynamique des $P M E$, de récentes études ont montré qu'en matière d'accès aux concours bancaires, ces firmes sont pénalisées en raison, d'une part, de leur taille et, d'autre part, de procédures d'évaluation du risque qui leurs seraient défavorables. L'objet de cet article est précisément d'examiner l'idée même de "risque PME" en tenant compte, premièrement, du degré d'intégration bancaire de l'activité de ces entreprises et, deuxièmement, de l'organisation industrielle dans laquelle elles s'inscrivent. Après avoir précisé les concepts de "risque de financement " et de "risque industriel", l'analyse montrera que le critère d'organisation industrielle constitue un critère pertinent de différenciation du risque de financement et du degré d'intégration bancaire de l'activité productive qui lui est associé. L'appréciation du risque de financement selon le critère de la taille apparaît dès lors particulièrement contre-productive.
\end{abstract}

* Christian Picory est maître de conférences à l'École nationale supérieure des télécommunications. Ses domaines de spécialisation sont: l'histoire de la pensée économique, l'économie industrielle (organisation industrielle, financement et théorie de la firme). Il est l'auteur de rapports d'études pour de grandes organisations publiques et privées, et d'articles consacrés aux PME (Revue Économique, Revue d'Économie Industrielle, Revue Internationale PME, etc.). Adresse : Département d'économie, 46, rue Barrault, 75634 Paris, cedex 13, France.

1. L'auteur remercie Luc Beal, Frédéric Chateau, Patrice Geoffron, Ludovic Lebart, ainsi que les deux évaluateurs anonymes de la Revue Internationale $P M E$, pour les commentaires et suggestions qu'ils ont bien voulu apporter aux versions préliminaires de ce texte (version 2 : février 1995 et version 3 : juin 1995. Il demeure bien évidemment seul responsable des éventuelles erreurs ou imprécisions qui pourraient subsister. 


\section{ABSTRACT}

While bank financing remains the principal method of financing the Small-andMedium-Sized firms dynamic, recent studies show that these firms are penalized because of their size and their risk evaluation procedures. The aim of this paper is to analyze the idea of "Small-and-Medium-Sized firms - Risk" by taking into consideration the variation in the level of integration of such companies with banks and the criteria derived from the industrial organization within which they operate. The author defines the concepts of "financing-risk" and "industrial-risk" and highlights the role of the industrial organization criteria in explaining the differentiation of "financing-risk" and the bank integration of the production activities related to it. The evaluation of "financing-risk" according to the size of firms criteria appears therefore counterproductive.

\section{RESUMEN}

Mientras que el financiamento bancario sigue siendo el principal modo de financiamiento de la pequeñas y medianas empresas (PyMEs), recientes estudios han demostrado que en materia de acceso a la asistencia bancaria, dichas firmas se ven penalizadas por una parte debido a su tamaño y por otra parte, a procedimientos de evaluación del riesgo que les scrian desfavorables. La finalidad de este articulo es precisamente examinar la idca misma del "riesgo PME" teniendo en cuenta en primer lugar el grado de integración bancaria de la actividad de esas empresas y en segundo lugar, la organización industrial en la cual se enmarcan. Después de especificar los conceptos de "riesgo de financiamiento" $y$ "riesgo industrial», el análisis mostrará que el criterio de organización industrial constituye un criterio pertinente de diferenciación del "riesgo de financiamiento " y del grado de integración bancaria de la actividad productiva que le está asociado. La apreciacion del riesgo de financiamiento según el criterio de tamaño resulta entonces particularmente contraproducente.

\section{Introduction}

Le mouvement d'innovation et de déréglementation des marchés financiers qui est intervenu en France au milieu des années 1980 a eu notamment pour effet de modifier les relations banques-entreprises. En s'appuyant sur le développement des marchés et sur les nouvelles stratégies de financement des sociétés, il a été possible de relever, d'une part, un certain rééquilibrage entre intermédiation bancaire et financement par le marché et, d'autre part, une profonde mutation de ces deux éléments eux-mêmes. Cette nouvelle intermédiation financière, qualifiée par certains d'économie de marché intermédiée, n'a pourtant pas répondu à tous les espoirs de ses promoteurs. Trois observations viennent en effet nuancer l'idée d'économie de marché intermédiée. 
Premièrement, les banques demeurent apporteurs nets de capitaux aux entreprises depuis le milieu de la décennie 1980. Le comportement financier des entreprises se caractérise par des emplois en capital qui dépassent les ressources dont elles bénéficient au titre des augmentations de capital. Le crédit bancaire reste donc le moyen de financement de la majorité des entreprises. Deuxièmement, les PME semblent avoir été écartées des possibilités offertes par la désintermédiation bancaire, la création du second marché n'apportant pas les résultats escomptés en termes de financement. Troisièmement, enfin, et en matière d'accès au financement bancaire, l'effet taille qui pénalise les PME-PMI reste important, en particulier pour les crédits à court terme et en dépit de leur réduction relative à la fin des années 1980 .

Au-delà d'un enrichissement qualitatif des relations banques-PME, consécutif au mouvement de désintermédiation bancaire du financement des grandes entreprises et perceptible en particulier au niveau de l'offre de services, les PME feraient donc face à la réalité d'un désengagement des banques dans leur rôle traditionnel de financier privilégié, alors même que le financement bancaire demeure la principale modalité de financement de leur dynamique. Diverses raisons sont avancées pour expliquer ce désengagement. Une part de l'écart des taux consentis aux firmes selon leur taille renvoie à un coût d'obtention de l'information plus important pour les PME ainsi qu'à des frais fixes qui sont très proches pour des dossiers dont les montants diffèrent sensiblement. Sont également avancés les perspectives incertaines liées à un très fort ralentissement de l'activité économique, les effets «pervers» de la loi Badinter $^{2}$ sur le redressement et la liquidation judiciaires, limitant le pouvoir des créanciers et, enfin, l'existence d'un risque objectivement plus élevé pour les PME. Sur ce dernier point, une étude de la Banque de France montre que le risque exerce un rôle sur la tarification des crédits (Bardos, 1990) - à taille égale, une entreprise à risque paiera un taux d'intérêt plus élevé -, mais ce rôle varie en fonction de la taille. Néanmoins, la hiérarchie des taux selon les risques ne recoupe pas celle des taux par taille d'entreprises. Les deux effets ne sont donc pas équivalents: la taille de l'entreprise exerce un effet propre indépendamment du risque de défaillance qui lui est lié. Il reste que ces éléments et, en particulier, le risque n'expliquent que partiellement l'écart des taux; ce qui conduit à supposer une mauvaise allocation des financements, un frein à l'investissement des PME solvables et, au contraire, un encouragement à celui des grandes entreprises à risques.

Diverses techniques sont envisagées pour améliorer l'accès des PME-PMI au crédit bancaire. Création d'une administration spécialisée (Small Business Administration aux États-Unis, par exemple), puissance du capitalisme familial

2. Loi 85-98 de janvier 1985. 
adossé à une banque principale (la Hausbank) comme en Allemagne, création de fonds de garantie. Au-delà de ces solutions plus institutionnelles, il est probable qu'une voie possible pour modifier cet état de fait, et pour prendre en compte des critères économiques plus pertinents dans l'appréciation du risque lié à l'activité des PME, serait de réduire, autant que faire se peut, l'asymétrie d'information. Dans cet esprit, certaines banques ont élaboré en interne un système d'information sur la rentabilité de la relation d'affaires avec les PME afin d'éclairer le prêteur. C'est qu'en effet l'évaluation des risques par des tiers ne paraît pas adéquate. Une enquête de l'AFB (limitée aux prêts indexés sur le taux de base bancaire qui concerne donc essentiellement les PME) a mis en évidence l'absence de liens étroits entre le coût du crédit et la cotation Banque de France; de même, les études sur la notation montrent que si les écarts de taux sont corrélés avec ceux des notes, les fluctuations de taux précèdent celles de la notation. Pour ces raisons, il est vraisemblable que les banques soient conduites à consacrer davantage de ressources pour une meilleure appréciation du «risque-PME».

Une évaluation directe peut être conduite, y compris pour des PME, grâce à la mise en place de cellules spécialisées pour des unités de production de taille réduite. La question est cependant de savoir si une approche statistique d'assurance combinant éventuellement l'utilisation d'une méthode de «scoring" et des fonds de garanties pourrait suffire. Il est probable que non, dans la mesure où une approche actuarielle peut entraîner une accumulation complémentaire de risques liés à la non-recherche d'information spécifique. En outre, d'autres formules envisageables, telles que l'intervention de Sofaris, et visant à mutualiser le risque, ne dispensent pas, en tout état de cause, de l'étude du dossier. Le système bancaire est donc placé devant la nécessité d'une meilleure appréciation du risque et une exploitation plus poussée des sources d'informations sur la vie des entreprises.

Dans le contexte ainsi précisé, notre contribution propose d'examiner l'idée même de «risque PME», sur lequel, nous l'avons vu, la taille exerce un effet propre indépendant de conditions structurelles que l'on peut supposer. Cet examen est effectué en liaison avec, d'une part, le degré d'intégration bancaire des firmes et, d'autre part, l'organisation industrielle dans laquelle elles s'inscrivent. Dans la perspective qui est la nôtre, l'analyse de la relation banque-PME, c'est-à-dire de son degré d'intégration bancaire, peut être structurée selon trois axes.

- l'engagement des banques dans le financement des investissements et de l'exploitation des PME;

- l'évolution de l'objet (conditions bancaires, information, garanties, etc.) et du pouvoir de négociation des PME face aux banques; 
- l'importance des financements non bancaires, pour lesquels les exigences de surface financière ou de garanties sont plus limitées.

L'analyse du «risque PME» sera effectuée dans le cadre d'une approche «contingente ${ }^{3}$ » de la relation banque-PME. À la suite des travaux de Coase (1937 et 1972), il apparaît en effet qu'une réflexion sur le risque et le degré d'intégration bancaire de l'activité de production et, plus généralement, sur la firme est indissociable de la nature de l'organisation industrielle ${ }^{4}$ dans laquelle elle s'inscrit. L'hypothèse fondamentale de ce travail est que le degré d'intégration bancaire de l'activité productive ne résulte pas uniquement des choix stratégiques en matière d'engagement opérés par les banques, en fonction des critères traditionnels de risque-rentabilité-solvabilité. Il dépend à titre principal des spécificités du contexte économique et social dans lequel s'inscrit la PME; ces spécificités définissant à leur tour divers niveaux de risque-rentabilité-solvabilité. Autrement dit, il existerait une relation entre la nature de l'organisation industrielle, définie en fonction de configurations particulières de caractéristiques de l'activité, et à laquelle appartiennent la PME, la nature, l'ampleur et l'intensité de l'intégration bancaire de l'activité productive et les divers niveaux de risque-rentabilité-solvabilité. Dans cet article, nous tenterons de préciser les configurations types en approchant les concepts de risque-rentabilité-solvabilité par l'intermédiaire du risque de défaillance.

Les résultats obtenus permettront alors, d'une part, de confirmer l'idée selon laquelle la taille de l'entreprise exerce un effet propre, mais limité dans l'appréciation du risque et, d'autre part, de préciser les critères économiques pertinents qui paraissent intervenir dans cette dernière. Dans cet esprit, nous préciserons en premier lieu le cadre théorique de l'étude, puis en second lieu la méthodologie de l'analyse. Nous interpréterons en troisième lieu les principaux résultats obtenus en nous fondant sur le concept d'organisation industrielle. Le couple risque-organisation industrielle s'articulera finalement avec le degré d'intégration bancaire de l'activité productive.

\section{Risque et organisation industrielle}

De manière générale, le risque individuel de l'entreprise comporte deux faces distinctes mais liées qui renvoient au circuit de la production (figure 1). Le circuit de la production est fondé sur deux axiomes fondamentaux (Cartelier,

3. Nous préciserons infra le concept de contingence auquel nous nous référons.

4. Dans l'immédiat et pour les seuls besoins de cette introduction, admettons que l'organisation industrielle recouvre la notion d'environnement. Ce concept sera précisé plus loin dans cet article. 
1991). Le premier tient à l'économie de marché elle-même. Dans le contexte d'une telle organisation des activités économiques, les agents (entreprises, consommateurs, etc.) ont besoin du marché pour coordonner leurs décisions, ce qui signifie qu'ils ne peuvent prévoir a priori et avec exactitude quel sera le résultat de la coordination. Ainsi, une entreprise ne peut-elle savoir ex ante si son nouveau produit trouvera un marché suffisant pour rentabiliser ses investissements. Tout au plus, les agents sont-ils en mesure de prévoir, de manière plus ou moins exacte, les résultats de la coordination. Le marché est donc un instrument de coordination a posteriori : ce n'est qu'en agissant au marché (échanges, achat de matières premières, ventes de produits, etc.) que les agents apprennent le degré de réalisation de leurs actions (validation marchande). Chaque agent est cependant libre de choisir son action (investir, embaucher du personnel, etc.) et est autonome. La rationalité des agents est cependant limitée. Les limites de la rationalité ne doivent toutefois pas être imputées à une absence de compétence, voire à une incohérence des acteurs. Elles sont précisément liées à l'autonomie des agents: c'est parce que chacun est autonome et que l'information nécessaire à la décision est, en grande partie, privée que le marché n'est pas transparent pour chacun de ses acteurs.

FIGURE 1

\section{Circuit de la production}

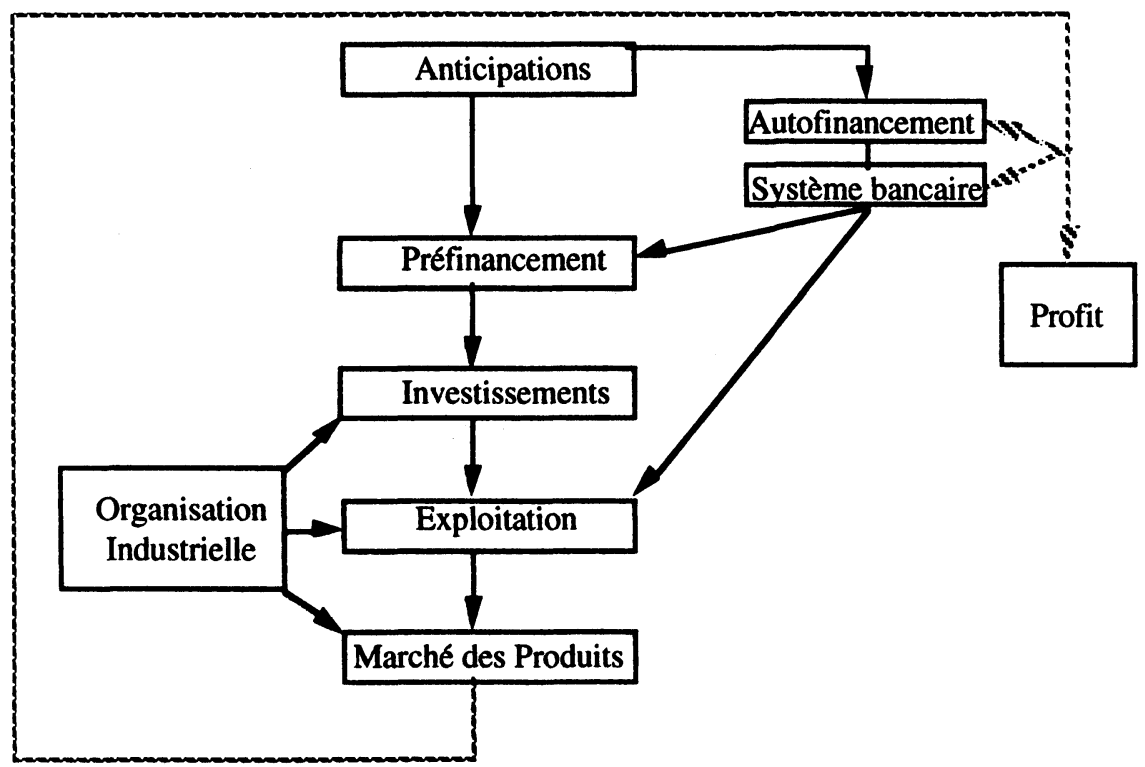

Source: C. Picory. 
Le second axiome fondamental concerne le système de paiement. Dans le cadre des économies de marché contemporaines, il faut retenir l'idée centrale que la monnaie est un moyen général de paiement, accepté par tous, indépendamment de toute autre considération. En d'autres termes, la monnaie est le moyen qu'ont les individus d'exécuter les actions qu'ils ont planifiées indépendamment de la constatation préalable du résultat d'une coordination a priori des activités économiques (préfinancement). Il en résulte, d'une part, un déphasage nécessaire dans la chronologie des flux de préfinancement et du reflux du chiffre d'affaires et, d'autre part, la possibilité pour n'importe quelle firme de se trouver effectivement en dehors de sa contrainte budgétaire, c'est-àdire de constater une différence entre la valeur réalisée de ses achats et de ses ventes. Cela se produit, non parce que cet agent est irrationnel, ayant prévu par exemple de ne pas tenir compte de sa contrainte de solvabilité (valeur des achats = valeur des ventes). La différence vient simplement de ce que l'exécution des plans individuels n'est pas soumise à une coordination a priori ou, ce qui revient au même, de ce que la rationalité des individus est limitée à l'élaboration des plans d'actions (anticipations) et ne peut s'étendre aux conditions de leur réalisation; cela pour diverses raisons liées, d'une part, au déphasage chronologique entre les flux de préfinancement et le reflux du chiffre d'affaires et, d'autre part, à l'organisation industrielle dans laquelle s'inscrit la firme, mais qu'en général elle ne contrôle pas totalement. Il en résulte que la compatibilité des plans individuels (les produits de l'entreprise correspondent-ils, par exemple, à la demande des consommateurs?) est sanctionnée par le marché et que cette sanction se traduit au niveau de la firme par deux contraintes fortes de solvabilité et de rentabilité (profit positif ou nul).

Du point de vue de la firme, le risque industriel est donc la non-validation ex post du projet prévu ex ante. Précisons cependant que ce risque ne renvoie pas à la notion classique de risque, c'est-à-dire à la notion d'événement aléatoire pour lequel existe une distribution objective de probabilités permettant au décideur de faire usage de cette information pour compenser les risques, au moyen de l'assurance, par exemple. Il renvoie plutôt à la notion d'incertitude, c'est-à-dire au cas où, soit les probabilités objectives sont inconnues de telle sorte que le décideur doit s'en remettre à une estimation subjective de la vraisemblance, soit au cas où les probabilités objectives sont éventuellement connues, mais où la situation présente par elle-même un caractère unique qui empêche de recourir à la compensation des risques (Knight, 1921). En dynamique, le risque industriel correspond au fait que le reflux n'est pas, pour un instant donné, suffisant pour honorer les engagements souscrits (risque de solvabilité) et peut être in fine inférieur à la valeur du préfinancement (risque de rentabilité). Ce risque est fondamentalement lié, d'une part, à l'autonomie des agents économiques et au caractère privatif de l'information et, d'autre part, au fait qu'entre anticipation et validation marchande du projet de production 
intervient l'organisation industrielle dans laquelle ce projet s'inscrit. Outre les facteurs classiques de contingence que sont la taille et le secteur d'activité, l'organisation industrielle renvoie aux cinq critères complémentaires suivants dont nous préciserons par quelques mots clés les contenus (Picory, 1994):

- caractéristiques du processus de production : formes de la spécialisation / division des activités productives (apprentissage, conception, production, commercialisation), échelle, standardisation, production orientée par la demande, etc. ;

- dynamique technologique : intensité et trajectoire technologique, performance des processus innovants, asymétries technologiques entre secteurs (innovations incrémentales / radicales), processus de diffusion de l'innovation entre firmes, innovation, économies d'échelle et organisationnelle ;

- formes des relations interfirmes : division du travail entre les firmes en liaison avec la spécialisation des activités, sous-traitance, relations marchandes, contractuelles ou informelles, coopération industrielle en matière de R-D, conception, production, commercialisation, relations d'intégration ;

- formes des relations de la firme avec son marché : formes du marché (acteurs, nature de la demande en variété et variabilité, marchés publics), dynamique concurrentielle (degré d'exposition à la concurrence nationale et internationale, concurrence prix ou hors prix);

- degré d'intégration bancaire de l'activité productive: nature et ampleur de l'engagement des banques dans le financement de l'activité (investissements et exploitation), objet (conditions bancaires, information, services, garanties) et pouvoir de négociation dans la relation banque-firme, asymétries d'information, nature et ampleur des financements non bancaires.

Sous l'hypothèse d'autonomie des firmes bancaires, d'une part, et industrielles, de l'autre ${ }^{5}$, le risque lié au financement bancaire (risque de financement) est de notre propre point de vue, différent bien que lié au précédent. Comme toute autre entreprise, les firmes bancaires sont soumises à la contrainte de rentabilité et de solvabilité. Les emplois inscrits à leur actif, et en particulier les créances sur le système productif, doivent dès lors contribuer à la marge nette bancaire et être recouvrées. Du point de vue de la banque, le non-recouvrement de créances ou leur recouvrement partiel s'interprète comme une nonvalidation du projet de «production» bancaire: les contraintes de rentabilité

5. Sur les conséquences de cette hypothèse sur les analyses d'économie industrielle du financement et de la firme bancaire, on pourra consulter Maricic et Picory (1991). 
et de solvabilité ne portent cependant que sur la part bancaire du préfinancement du projet industriel. De manière générale, risque de financement et risque industriel ne se confondent pas au sens où ils renvoient à deux catégories différentes d'agents et donc à deux logiques qui n'ont aucune raison de converger, bien qu'elles soient soumises aux mêmes contraintes générales.

- Dans le cadre d'une logique bancaire «à l'acte» (banque fournisseur de la firme $)^{6}$, la relation avec la firme s'appuie sur le triptyque risque-rentabilité-solvabilité. Le risque est alors perçu par la banque comme un événement aléatoire qui s'inscrit dans un cadre probabiliste objectif et l'analyse du risque renvoie, d'une part, aux techniques de l'actuariat et, d'autre part, à l'évaluation du risque individuel selon des procédures standardisées à validité générale (scoring, analyse financière classique). Le contenu de la relation banque-firme, qu'il s'agisse de la réduction des asymétries d'information, de l'imposition de nombreuses garanties ou encore de la forme des concours, a pour seul objectif la minimisation du risque assumé par la banque 7 . Risque industriel et risque de financement sont cependant liés dans la mesure où le financement bancaire constitue une part non négligeable du préfinancement des projets de production.

- La logique industrielle relève de l'organisation industrielle et renvoie de manière générale aux formes d'organisation des processus de production, à la dynamique technologique, aux formes des relations entre les firmes et, enfin, aux structures, comportements et performances des firmes sur les marchés. Dans ce contexte, si la finance constitue une contrainte en amont (préfinancement), dès que les investissements prévus sont réalisés, elle ne représente qu'une variable d'ajustement ex post par l'intermédiaire du financement bancaire de l'exploitation (découvert bancaire, crédit global d'exploitation) ${ }^{8}$.

6. Sur ce point, $c f$. Rivaud-Danset (1991).

7. Cette logique bancaire s'oppose à une logique de «l'engagement» (banque partenaire de la firme). Cette dernière est fondée sur une relation de long terme avec la firme. Par une collaboration étroite avec cette dernière, la banque prend en charge une partie de l'incertitude radicale assumée par l'entreprise et accompagne cette dernière dans la réduction des risques et leur gestion en lui fournissant des services d'information et de conseils. A contrario, dans le cadre du modèle de l'acte (banque fournisseur de la firme), les relations banque-firme relèvent plus du court terme et s'apparentent plus au simple contrat d' «échange» anonyme qu'à une relation de coopération.

8. Cela ne signifie pas que le financement bancaire de l'exploitation soit négligeable. Il nous paraît, tout au contraire, essentiel dans la définition du degré d'intégration bancaire de la firme. Nous reviendrons sur cet aspect fondamental dans la section 4 de ce texte. 
Il existe toutefois deux cas exceptionnels pour lesquels risque industriel et risque de financement convergent. Le premier est celui où justement la distinction entre les deux types de risque tend à s'atténuer: il s'agit alors du modèle d'intégration banque-industrie, incompatible cependant avec l'idée d'autonomie des deux catégories d'acteurs. Le second concerne la situation de défaillance de l'entreprise, c'est-à-dire le moment à partir duquel une procédure de redressement judiciaire est ouverte à son encontre, en raison de son incapacité de faire face à son passif exigible avec son actif disponible (cessation de paiement). Dans ces conditions, il est clair que l'entreprise est, sur l'ensemble de ses projets de production en cours, en dehors de ses contraintes de rentabilité et de solvabilité tandis que la banque est, pour les seuls projets qu'elle a cofinancés, également en dehors de ces contraintes. Cette situation exceptionnelle, où risque de financement et risque industriel convergent, justifie l'analyse de la liaison organisation industrielle-risque de défaillance.

\section{Méthodologie de l'étude}

En cas de cessation de paiements, l'entreprise est tenue de déposer son bilan au greffe du tribunal de commerce compétent qui prononce un jugement soit de liquidation ${ }^{9}$, soit de redressement (continuation de l'activité ou reprise). Comme tous les jugements, celui-ci doit faire l'objet d'une publication dans le Bulletin officiel des annonces civiles et commerciales (BODACC), dont le traitement permet d'élaborer les statistiques de défaillances d'entreprises. Ces données seront rapprochées, pour l'année 1993, au stock d'entreprises issu du fichier SIRENE, répertoire des entreprises géré par l'INSEE. Les cessations enregistrées dans ce fichier ne recouvrent cependant pas les seules défaillances, au sens où il comptabilise, outre les cas de disparition à la suite d'une procédure de redressement judiciaire, les modifications de statut d'entreprises, les fermetures volontaires, les cessions et les fusions-acquisitions, juridiquement interprétées comme disparitions d'entreprises. Par ailleurs, le stock d'entreprises comporte également les créations, dont les variations affectent également le dénominateur d'un taux de défaillance. En dépit de ces deux difficultés, qui impliquent une interprétation nuancée des taux, nous restreindrons l'étude des défaillances d'entreprises aux secteurs industriels pendant l'année 1993. Cette restriction se justifie pour deux raisons.

La première tient à la disponibilité des données. L'analyse qui vient s'appuie en effet sur des statistiques de défaillances par tailles et par activités, selon la nomenclature d'activités et de produits (NAP 73) au niveau 600. Or, ces données ne sont disponibles que pour 1993.

9. Il s'agit de $95 \%$ des cas, selon le ministère de la Justice. 
La seconde raison relève du phénomène des défaillances lui-même. Globalement, en effet, celles-ci concernent des catégories particulières de firmes (Blazy, Charlety et Combier, 1993; Combier, 1994).

- Les entreprises les plus jeunes, tout d'abord : $55 \%$ des entreprises défaillantes en 1993 étaient âgées de moins de cinq ans, alors que les entreprises de cette tranche d'âge ne représentaient que $38 \%$ du stock au $1^{\text {er }}$ janvier 1993. Le taux de défaillance le plus élevé concerne les entreprises de 2 à 3 ans (6\%), tandis que les classes d'âge extrêmes (moins de un an et plus de 10 ans) paraissent moins affectées.

- Les petites PME, ensuite. Les taux de défaillance les plus faibles concernent les classes de taille extrêmes : 0 ou 1 salarié, d'une part, et plus de 100 salariés, de l'autre $(2,9 \%$ et $2,4 \%)$. Les taux les plus élevés peuvent être observés pour les PME de 10 à 19 salariés $(5,4 \%)$, de 20 à 99 salariés $(5 \%)$, puis de 5 à 9 salariés $(4,6 \%)$.

- Les entreprises industrielles, enfin. Les taux de défaillance les plus élevés concernent le commerce de gros $(4,8 \%)$, l'industrie $(4,5 \%)$ et le BTP $(4,4 \%)$. Les salariés touchés par les défaillances sont toutefois relativement plus nombreux dans l'industrie que dans les autres secteurs. En effet, si les entreprises de l'industrie (biens de consommation, biens intermédiaires et biens d'équipement) et du BTP représentent $37 \%$ (c'est-à-dire respectivement $16,9 \%$ et $19,9 \%$ ) de l'ensemble des entreprises défaillantes, elles représentent $58 \%$ (c'est-àdire respectivement $38,8 \%$ et $18,7 \%$ ) des effectifs salariés concernés par les défaillances d'entreprises.

Afin d'examiner les défaillances d'entreprises industrielles ${ }^{10}$ en fonction des spécificités de leur activité, nous avons tout d'abord défini plus finement la notion d'activité en utilisant le concept d'organisation industrielle présenté plus haut. Parmi les critères présentés, nous avons choisi de porter l'accent sur les critères relevant plutôt de l'organisation externe des PME, leur degré d'intégration bancaire constituant une variable illustrative. Comme indiqué plus haut, l'analyse doit intégrer la frontière de la firme, c'est-à-dire son positionnement dans un «environnement » particulier, au titre duquel il faut inclure les caractéristiques du processus de production (échelle, standardisation, production à la demande, production spécialisée, etc.), le niveau d'intensité technologique et de performance des processus innovants, les caractéristiques

10. En vertu des restrictions explicitées supra et par rapport à la NAP 600, l'industrie sera définie par agrégation des niveaux : 10,11,13,14,15.12,15.13,16,17, 18, $19,20,21,22,23,24,25,26,27,28,29,30,31,32$ (à l'exclusion de 32,05 ), 33 , $34,43,44,45,46,47,48,49,50,51$ (à l'exclusion de 51,01 ) ; 52,01;52,03; 53 ; 54 (à l'exclusion de 54,09 et 54,10 ). 
et le niveau d'expansion de la demande, le degré d'exposition à la concurrence (sur un marché national ou international), l'inscription de la firme dans un rapport de sous-traitance ou encore le soumissionnement aux marchés publics.

Le rôle de certains de ces facteurs dans la structuration de l'appareil productif a fait l'objet de travaux effectués dans le cadre du SPRU (Science Policy Research Unit) de l'Université du Sussex. Ceux-ci ont permis de proposer une nouvelle nomenclature des secteurs industriels en liaison avec les caractéristiques précédentes et, en particulier, avec les processus d'innovation technologique (Dosi, Pavitt et Soete, 1985; Pavitt, 1984). En partant de ces caractéristiques et en reformulant, sur cette base, la notion de secteur, il a été possible de définir une typologie de PME permettant une analyse plus fine de la dynamique de ces firmes et de leurs stratégies (Picory, 1990a et b; Picory, 1994). Cette typologie comporte quatre «secteurs» ou organisations industrielles.

1. Les secteurs caractérisés par une intensité élevée de recherche de base (science based). Nous les noterons $S B$ pour la suite du texte. Ces secteurs sont habituellement générateurs nets d'innovation technologique. Ils sont caractérisés par une forte activité de recherche de base que ce soit au niveau de l'entreprise ou encore de structures plus larges telles que les technopoles, une lente «gestation» des projets innovants opposée à un cycle de vie des produits très rapide, une très forte dépendance aux structures de recherche publiques ou privées, une faible sensibilité aux facteurs de compétitivité-prix. La soustraitance, enfin, en est une de "spécificité»: le donneur d'ordres externalise une production pour laquelle il ne dispose pas de la maîtrise du métier. Le cahier des charges n'est alors pas standard, normé, mais fait l'objet d'une concertation. Des exemples classiques peuvent être trouvés parmi les industries des télécommunications, des composants actifs, de l'aérospatiale, de l'informatique, des nouveaux matériaux de synthèse, des diverses applications des biotechnologies.

2. Les secteurs caractérisés par une dimension organisationnelle élevée (scale intensive). Nous les noterons SI pour la suite du texte. Ces secteurs sont caractérisés par des productions à haut volume et une grande flexibilité de gamme, une intense activité de recherche appliquée aux innovations de procédés, une forte propension à incorporer des innovations-produits issues d'autres secteurs, une dynamique de l'innovation de procédés ou organisationnelle contrainte par une grande sensibilité aux coûts des inputs (énergie, travail, matières premières), ainsi qu'à la concurrence sur les prix relativement plus élevée, de fortes dépenses en liaison avec la publicité, le marketing, les services après-vente. Les industries automobile, de l'électroménager «blanc», 
ainsi qu'une large part des industries de la pharmacie, constituent quelques exemples de secteurs scale-intensive.

3. Les secteurs orientés par la demande (specialized suppliers). Nous les noterons $S S$ pour la suite du texte. Ceux-ci sont caractérisés par une grande flexibilité de leur production par rapport aux exigences spécifiques de leurs clients, qu'il s'agisse d'utilisateurs intermédiaires ou finaux, une prévalence des entreprises de taille relativement limitée, une spécialisation élevée sur des productions particulières ou dans le cadre de «niches » technologiques restreintes, une très forte concurrence hors prix (non price competition) sur la flexibilité, la fiabilité et la qualité des produits, les capacités d'adaptation et d'assistance. La sous-traitance réalisée par les firmes de ces secteurs en est une de spécialité : le donneur d'ordres externalise une production pour laquelle il dispose de la maîtrise du métier. Le cahier des charges est alors normé et l'autonomie du sous-traitant plus réduite. Des exemples aujourd'hui classiques peuvent être trouvés parmi les industries de la mécanique de précision, des instruments de mesure, de l'appareillage biomédical, etc.

4. Les secteurs traditionnels, notés ST. Ces derniers sont utilisateurs nets d'innovations de produits et de procédés développées dans les autres secteurs et mobilisent relativement peu de moyens en faveur du poste R-D. Comme pour les secteurs caractérisés par une dimension organisationnelle élevée $(S I)$, les innovations de procédés s'inscrivent dans le cadre d'une stratégie de réduction des coûts. L'environnement concurrentiel est non seulement déterminé par les facteurs prix, mais aussi par des facteurs hors prix tels que le design, la qualité, la fiabilité, la variété de gamme, la compétence des services marketing et des réseaux de distribution commerciale. La sous-traitance assurée par les firmes de ces secteurs en est une de capacité. En exemple, nous citerons les industries de l'habillement (prêt-à-porter, chaussure, textile, cuirs et peaux, etc.), de l'ameublement, du jouet et des instruments de musique.

Cette typologie, transversale aux taxonomies usuelles, est fondée sur des caractéristiques de l'offre et de la demande qui conduisent à une composition de secteurs extrêmement diversifiée, si nous ne retenons que le concept classique de produit homogène: il suffit de reprendre, pour chacun d'entre eux, la liste des exemples pour s'en convaincre. Elle constitue cependant un outil permettant de saisir la place qu'occupent les PME dans la structure de l'appareil productif et, par suite, de comprendre leur rôle dans la structuration de l'offre nationale. 
À partir des indications qui précèdent et en nous fondant sur des études antérieures, nous avons alors ventilé les 213989 entreprises industrielles en fonction de l'activité supposée homogène et repérée par le code APE, dans les quatre secteurs (ou catégories d'organisation industrielle).

\section{Défaillance d'entreprises et organisation industrielle}

Dans les pages qui viennent, nous chercherons à tester l'hypothèse suivante:

$\boldsymbol{H}$ : Il existerait une relation entre la nature de l'organisation industrielle, définie en fonction de configurations particulières de caractéristiques de l'activité, à laquelle appartient la PME, la nature, l'ampleur et l'intensité de l'intégration bancaire de l'activité productive et les divers niveaux de risque (de financement)-rentabilité-solvabilité.

La relation existant entre risque de financement, approximé par le taux de défaillance et l'organisation industrielle, peut être examinée à partir des données contenues dans le tableau suivant.

\section{TABLEAU 1}

Taux de défaillance

\begin{tabular}{lccc}
\hline Secteurs & $\begin{array}{c}\text { Taux } \\
\text { de défaillance }\end{array}$ & $\begin{array}{c}\text { Poids } \\
\text { dans l'industrie }\end{array}$ & $\begin{array}{c}\text { Part des défaillances } \\
\text { dans l'industrie }\end{array}$ \\
\hline ST & 4,8404 & 47,43 & 51,20 \\
SS & 4,429 & 26,28 & 25,96 \\
SI & 4,0548 & 16,86 & 15,24 \\
SB & 3,6201 & 9,42 & 7,61 \\
INDUSTRIE & 4,4848 & 100 & 100 \\
\hline
\end{tabular}

Sources : BODACC, INSEE et C. Picory.

Deux observations principales peuvent être tirées de l'analyse de ces données.

La première tient aux différences observées ainsi qu'à l'ordre introduit entre les différents secteurs. Contre toute attente ${ }^{11}$, le risque de financement tend à décroître avec l'intensité et les performances des processus innovants. Il convient toutefois de s'interroger sur le degré de signification de ce résultat.

11. Contre toute attente, dans la mesure où les processus innovants, qu'il s'agisse des innovations de produits, de procédés ou de l'organisation, peuvent être considérés, a priori et en dehors de toute analyse, comme une activité plus risquée qu'une activité traditionnelle. 
À cet effet, nous avons effectué un test de comparaison des taux de défaillance pour chaque paire de secteurs. Nous avons interprété la part des défaillances comme réalisation d'une variable aléatoire $F$. En désignant respectivement par $p_{1}$ et $p_{2}$, les parts de défaillances, dans les secteurs $S_{1}$ et $S_{2}$, il s'agit de savoir s'il existe une différence significative entre les deux proportions inconnues $p_{1}$ et $p_{2}$, au vu des fréquences observées $f_{i}$ d'échantillons aléatoires de tailles $n_{i}$ des secteurs $S_{i}$. Le test s'articule autour de l'hypothèse:

$$
\mathrm{H}_{0}: p_{1}-p_{2}=0
$$

Si cette hypothèse est vraie, alors la loi de probabilité de $\left(f_{1}-f_{2}\right)$ est une loi normale de moyenne 0 et d'écart type $\sqrt{f(1-f)\left(\frac{1}{n_{1}}+\frac{1}{n_{2}}\right)}$ et la variable $t$ définie par $|t|=\frac{\left|f_{1}-f_{2}\right|}{\sqrt{f(1-f)\left(\frac{1}{n_{1}}+\frac{1}{n_{2}}\right)}}$ suit une loi normale centrée réduite. Pour un seuil de risque $\alpha, \mathrm{H}_{0}$ peut être rejetée si $|t| \geq t_{\alpha}$. Les résultats des différents tests sont fournis au tableau 2.

Ces résultats montrent que $\mathrm{H}_{0}$ peut être rejetée, pour des seuils de risque très significatifs, en nuançant légèrement pour la comparaison $S I / S S$ pour laquelle le seuil de risque vaut $1,6 \%$, seuil très acceptable cependant.

La deuxième observation est liée à une question. Il convient en effet de s'interroger sur l'existence d'un effet-taille implicite auquel renverrait l'effet «d'organisation industrielle» qui est observé. Les classes d'entreprises les moins vulnérables au risque de défaillance (c'est-à-dire 0,1 et plus de 100 salariés) ne sont-elles pas surreprésentées précisément dans les catégories d'organisation industrielle (les «secteurs»), les moins exposées au risque de défaillance?

TABLEAU 2

Tests de comparaison des taux de défaillance

\begin{tabular}{llllllll}
\hline $\boldsymbol{S}_{\mathbf{1}}$ & $\mathbf{S}_{\mathbf{2}}$ & $\boldsymbol{f}_{\mathbf{1}}$ & $\boldsymbol{f}_{\mathbf{2}}$ & $\boldsymbol{n}_{\mathbf{1}}$ & $\boldsymbol{n}_{\mathbf{2}}$ & $|\boldsymbol{t}|$ & Probabilité critique \\
\hline$S B$ & $S I$ & 0,036 & 0,04 & 20165 & 36081 & 2,3626035 & $\approx 9,1.10^{-3}$ \\
$S B$ & $S S$ & 0,036 & 0,044 & 20165 & 56243 & 4,865164 & $\approx 10^{-6}$ \\
$S B$ & $S T$ & 0,036 & 0,048 & 20165 & 101500 & 7,4289528 & $<10^{-9}$ \\
$S I$ & $S S$ & 0,040 & 0,044 & 36081 & 56243 & 2,9418555 & $\approx 1,6.10^{-2}$, \\
$S I$ & $S T$ & 0,040 & 0,048 & 36081 & 101500 & 6,2369299 & $<10^{-7}$ \\
$S S$ & $S T$ & 0,044 & 0,048 & 56243 & 101500 & 3,6110857 & $<10^{-3}$ \\
\hline
\end{tabular}

Source: C. Picory. 
Cette hypothèse nous apparaît cependant hautement improbable. En effet, la figure 2 montre que les secteurs $S T$ et $S S$, les plus exposés au risque de défaillance, comportent significativement plus d'entreprises faiblement vulnérables (au sens de la taille) que les secteurs SI et SB. De même, les secteurs $S I$ et $S B$, les moins vulnérables, paraissent comporter plus d'entreprises moyennement exposées au risque de défaillance. Enfin, les quatre secteurs sont composés d'une proportion oscillant entre 17 et $19 \%$ d'entreprises appartenant aux catégories les plus exposées au risque de défaillance (10 à 19 ou 20 à 99 salariés). Cet effet de contre-structure, particulièrement observable pour les secteurs $S T$ et $S S$, permet dès lors de rejeter l'idée d'un effet-taille.

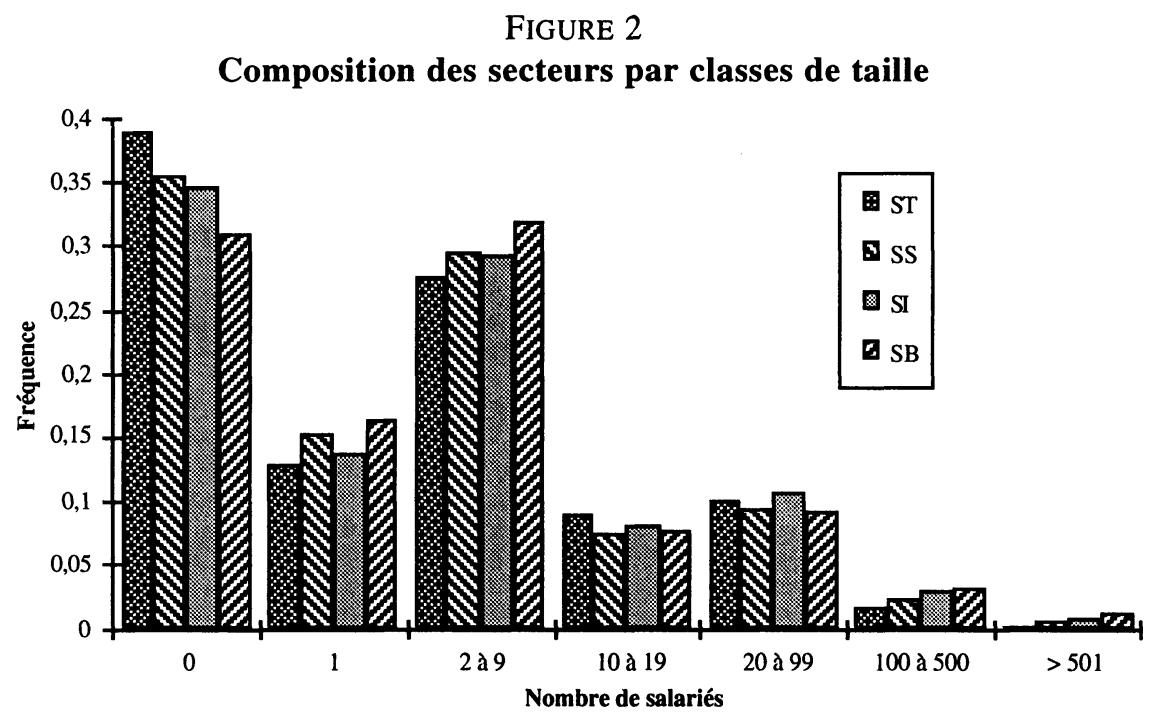

Sources : BODACC, INSEE et C. Picory.

La relation observée pourrait également témoigner des effets sélectifs exercés par le système bancaire, surtout pour les entreprises innovantes. Dans cet esprit, seuls les projets minimisant le risque de financement seraient alors sélectionnés, de telle sorte qu' in fine, les entreprises où l'intensité des processus innovants est la plus forte présenteraient les taux de défaillance les plus faibles. S'il est possible d'admettre une analyse bancaire du risque de financement plus fine en ce qui concerne ce type de firmes, l'argument nous paraît cependant devoir être écarté dans la mesure où il ne permet pas d'interpréter les différences observées dans les autres catégories d'organisation industrielle. Un raisonnement identique permet également de rejeter les effets très sélectifs que produiraient les aides et subventions de toutes natures, destinées aux entreprises les plus innovantes. 
En dépit du fait que l'effet «âge de l'entreprise» sur les secteurs n'ait pu être testé, en raison de la non-disponibilité des données, les développements précédents tendent à accréditer l'idée selon laquelle il existerait une corrélation entre la nature de l'organisation industrielle dans laquelle s'inscrit la firme et le risque de financement que nous avons approché par les taux de défaillance. À cet égard, en comparant les caractéristiques essentielles de chaque secteur et en appuyant les différences, la corrélation observée nous paraît relever de deux effets (figure 2). D'une part, un effet «massif» qui tend à lier, en raison inverse et contre toute attente, l'intensité et les performances des processus innovants et le taux de défaillance (corrélation très significative) et, d'autre part, un effet «de nuance» qui tend à lier en raison directe le taux de défaillance et le degré d'exposition à la concurrence; l'effet de nuance provenant du fait que degré d'exposition à la concurrence et intensité de la R-D ne sont probablement pas des variables indépendantes ${ }^{12}$. La combinaison des deux effets tendrait alors à définir des niveaux de risque objectivement différenciés, selon la nature de l'organisation industrielle.

\section{FIGURE 3}

\section{La relation défaillance - organisation industrielle}

Forte innovation procédé-produit-organisationnelle

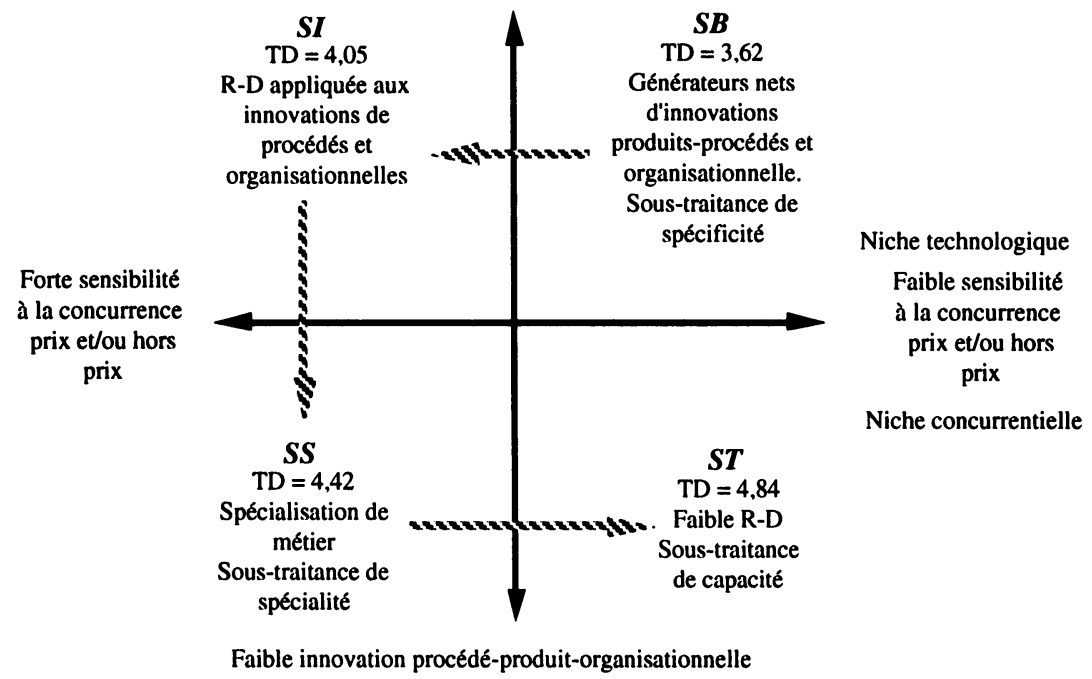

Source: C. Picory.

TD : Taux de défaillance

12. Une liaison inverse pourrait en effet être supposée. 
Il y aurait donc contingence du risque de défaillance par rapport à cette dernière. Notons cependant que le type de contingence examinée procède d'une approche statistique. Cela signifie que les entreprises appartenant à telle ou telle catégorie présentent en moyenne des caractéristiques affirmées et statistiquement discriminantes pour des seuils de risque définis. Cela n'exclut nullement la variance par rapport à la moyenne qui renvoie non seulement à d'autres facteurs explicatifs, de plus faible poids cependant, mais encore au comportement du chef d'entreprise. Il n'y a donc pas déterminisme. Et si notre approche procède d'un individualisme méthodologique, ce dernier est largement contextualiste.

En dépit du fait que la proposition qui précède mériterait une analyse beaucoup plus fine, mais qui dépasse la problématique définie pour cette étude, les résultats obtenus suscitent immédiatement une question. La liaison risqueorganisation industrielle ne témoignerait-elle pas de l'inégale capacité des entreprises de mobiliser les ressources, bancaires en particulier, nécessaires à leur dynamique?

\section{Défaillance d'entreprises et degré d'intégration bancaire}

Afin de comprendre le poids inégal des contraintes d'accès aux ressources de financement, le raisonnement pourrait être le suivant. En théorie, l'accès au crédit bancaire, de même que la différenciation des taux d'intérêt ne dépend pas seulement du montant, de l'échéance des prêts, mais aussi des caractéristiques du débiteur individuel. En théorie toujours, la seule caractéristique pertinente est le risque de défaillance. Mais en pratique, l'évaluation de celui-ci est difficile et son coût, sensiblement fixe, devient prohibitif en termes relatifs pour les opérations de faible montant. Les créanciers substituent alors, à une évaluation individualisée du risque, une estimation forfaitaire, à laquelle serait ajoutée, en raison d'un comportement prudentiel, une marge d'erreur représentative de la variabilité du risque au sein de la population considérée. Cette explication reporterait alors le critère d'accès au crédit bancaire, comme la différenciation du coût du crédit sur le montant des prêts sollicités ainsi que, implicitement au moins, sur l'appartenance à une organisation industrielle spécifique et non plus sur l'analyse du risque individuel de défaillance.

Cette argumentation n'a pu être testée directement sur la population étudiée, en raison de la non-disponibilité des données. Il est toutefois possible de l'illustrer, et seulement de l'illustrer, à partir des données d'une enquête réalisée en octobre et novembre 1993, sur un échantillon de 102 PME industrielles, issues d'une population de base de 800 PME du fichier de la Chambre de commerce et d'industrie de Paris. L'objet de cette enquête était d'analyser 
la dynamique des PME, ainsi que d'expliciter les contraintes, financières et, en particulier, bancaires qui pèsent sur cette dernière. En regroupant les PME dans les quatre catégories d'organisation industrielle, selon la méthodologie exposée supra et en concentrant l'analyse sur l'examen des relations des PME avec le système bancaire, le traitement des informations recueillies a permis de mettre en évidence trois degrés d'intégration bancaire de l'activité des PME (Picory et Geffroy, 1994 et 1995).

\subsection{Classe des PME SB, dont le degré d'intégration bancaire est élevé, l'activité est risquée, mais non contrainte par le système bancaire}

Compte tenu de l'importance de la recherche de base et de l'évolution technologique dans la dynamique des PME $S B$ et de la relative protection qu'assure l'avantage technologique par rapport aux contraintes concurrentielles classiques, $1^{\prime}$ 'indice ${ }^{13}$ de contrainte de dynamique de marché (marché incertain, niveau de la concurrence) est très inférieur à la moyenne $(-2,46)^{14}$. Si l'investissement ne paraît pas sensible aux contraintes de rentabilité et de structure financière (indices très inférieurs à la moyenne: respectivement $-7,22$ et $-4,41$ ), les contraintes de taux d'intérêt et de risque bancaire (conditions de financement et niveau des garanties) semblent affecter particulièrement la dynamique de ces entreprises (indices de contraintes très sensiblement supérieurs à la moyenne: respectivement 8,52 et 2). Ce «profil» nous paraît devoir être lié au degré d'intégration bancaire élevé de l'activité de ces firmes.

13. Sur la signification de ces indices, on se reportera à l'annexe.

14. Le nombre entre parenthèses indique une valeur test. Nous ne retiendrons que des valeurs tests supérieures ou égales à 1,66 en valeur absolue. Cette borne correspond à un risque de première espèce inférieur ou égal à $5 \%$, c'est-à-dire à une probabilité d'erreur inférieure ou égale à $5 \%$ en cas de rejet de l'hypothèse d'indépendance entre les deux variables. Pour les variables nominales, nous ne retiendrons dans toutes les caractérisations qui suivent que des différences entre le pourcentage des firmes d'une classe vérifiant une modalité donnée et le même pourcentage, mais sur l'ensemble de l'échantillon, substantiellement significatif. Autrement dit, nous ne nous fonderons, en règle générale, que sur des écarts entre la première et la seconde grandeur, tels que la probabilité pour que cet écart soit dû au hasard est inférieure à $5 \%$, ce qui correspond à une valeur test supérieure ou égale à 1,66 en valeur absolue. Lorsque la valeur test est positive, la modalité est caractéristique des individus de la classe; dans le cas contraire, elle ne saurait les caractériser. Pour de rares cas, des valeurs tests légèrement inférieures seront mentionnées, mais uniquement lorsque les modalités auxquelles elles se réfèrent confirment des corrélations statistiques établies selon la règle précédente. Dans les développements qui suivent ne seront indiquées que les valeurs tests. 
Pour ces firmes, en effet, la part de l'endettement dans les fonds stables est très supérieure à la moyenne $(1,73)$ et le niveau des fonds propres est jugé suffisant $(3,27)$; ce qui laisse supposer une surface financière importante dans la mesure où ces PME prévoient une augmentation de leur endettement dans les fonds stables $(6,86)$. Corrélativement, les parts du leasing-crédit bail et de l'autofinancement dans le financement des investissements sont, respectivement, très inférieure à la moyenne $(-5,48)$ et inférieure à la moyenne $(-1,84)$. En matière de concours bancaires à court terme, le degré d'intégration est tout aussi significatif: le crédit global d'exploitation finance souvent l'activité $(6,85)$ et les crédits en devises sont souvent utilisés $(3,97)$ lorsque la firme est exportatrice ${ }^{15}$. Le crédit interentreprises complète souvent $(3,20)$ ces concours. Le système bancaire assure donc une part considérable du financement de l'exploitation, de telle sorte que l'occurrence de problèmes de trésorerie est jugée sans importance $(2,64)$. Enfin, les conditions de financement sont rarement négociées sauf, de manière significative, pour les taux d'intérêt appliqués aux emprunts $(3,80)$.

Ces résultats nous permettent alors d'expliquer le poids des contraintes de garantie et de taux sur les investissements des PME SB. En effet, Bardos (1990) a remarqué que le taux d'intérêt apparent s'accroît avec le risque de défaillance (pour toutes les classes de taille d'entreprises) et que l'augmentation de l'endettement et la déformation de sa structure (au profit du court terme) accompagnent l'aggravation du risque. Si l'on admet que, du point de vue du banquier, l'activité des $S B$ est a priori risquée ${ }^{16}$ (risque éventuellement accru par l'intégration bancaire forte à court et long terme), on peut alors imaginer que le système bancaire applique au taux d'intérêt une prime de risque catégorielle liée à l'activité de l'entreprise ${ }^{17}$, et accroisse ses exigences en matière de garanties. La sensibilité de la dynamique d'investissement des $S B$ par rapport à l'évolution des taux d'intérêts (et son impact sur le taux de base bancaire) et des conditions de garanties est alors plus grande. Cette sensibilité n'entame cependant pas l'évaluation de la performance bancaire par les PME $S B$.

De manière très significative, ces firmes sont en relation avec une banque unique $(3,80)$ et $n$ 'envisagent pas d'en changer $(2,90)$. Cette fidélité ne renvoie pas à la participation de la banque au capital de l'entreprise $(3,85)$ ou à sa création $(2,66)$, mais à la qualité de la relation. Les professionnels de la banque concernés sont soit des directeurs d'agence de banques spécialisées

15. En moyenne, ces PME réalisent de 1 à $19 \%$ de leur CA à l'exportation $(4,8)$.

16. Cela bien que le taux de défaillance pour ces firmes soit significativement le moins élevé. Y aurait-il une distorsion de taux pour les firmes SB?

17. Éventuellement complétée d'une prime de risque dépendant de la situation individuelle de l'emprunteur. 
dans les PME $(3,83)$, soit des directeurs spécialisés dans les PME, lorsque la banque est plus généraliste. Dans le cadre de l'EDI (échange de données informatisées), les flux d'information entre PME et banque sont équilibrés, ce qui laisse supposer de faibles asymétries d'information entre les acteurs, bien que la transmission d'information sensible sur l'entreprise soit «modérée» $(1,66)^{18}$. Ce dernier point renvoie davantage au très fort degré de confiance mutuelle établie entre les partenaires $(2,20)$, ainsi qu'à l'autonomie de la firme en matière de stratégie à long terme ou de gestion courante, qu'à une défiance de la firme par rapport à la banque. Dans une étude antérieure (Fernandez, Picory et Rowe, 1993), nous avons en effet montré que les firmes $S B$ effectuent une planification de leur activité dans le cadre d'un modèle de régulation ex ante de l'activité: la rationalisation de l'activité est prévue et le pilotage boucle sur celle-ci; les formes de la concurrence et de la dynamique technologique sont anticipées et permettent un contrôle proactif des relations de la firme avec son environnement. L'EDI bancaire ainsi que la forte collaboration avec la banque s'inscriraient alors dans cette logique. Dans ces conditions, l'indice de satisfaction de la fonction bancaire «compréhension globale de la situation de la PME» est très supérieur à la moyenne observée pour les PME $S B(5,82)$. Il en est de même pour l'indice de satisfaction par rapport aux fonctions bancaires traditionnelles (aide de la banque pour la gestion de la trésorerie et la gestion des placements, financement bancaire des investissements) (2,81), par rapport à la gestion du risque (aide de la banque pour la gestion du risque client et du risque à l'exportation) (1,98). Compte tenu de l'autonomie de la firme dans ses choix stratégiques ou dans sa gestion courante, l'indice synthétisant la performance de la banque en matière de conseils (gestion courante, stratégie à long terme, marchés extérieurs) ne ressort pas de manière significative. Au total, l'indice de satisfaction synthétique est très significativement supérieur à la moyenne $(5,65)$.

\subsection{Classe des PME SI dont le degré d'intégration bancaire est élevé, mais dont l'activité relève de marchés dynamiques}

Bien qu'exposées aux contraintes concurrentielles classiques, les firmes de cette classe affichent une forte insensibilité par rapport aux contraintes de dynamique de marché ou de rentabilité, ce qui témoigne de leur dynamisme sur leurs marchés ${ }^{19}$ : à l'appui de cette idée, relevons les niveaux élevés de

18. Dans cet esprit, l'EDI découlerait non seulement d'une procédure de réduction du coût bancaire de la gestion des moyens de paiements, mais encore d'une problématique de réduction des asymétries d'information banque-entreprises.

19. Pour ces PME, l'indice de contrainte de dynamique de marché est très sensiblement inférieur à la moyenne $(-8,02)$. Il en est de même pour l'indice de contrainte de rentabilité $(-14,79)$. 
rentabilité $(8,04)$, ainsi qu'une anticipation de croissance de celle-ci $(3,92)$. Ces faits sont à rapprocher, d'une part, des stratégies de réduction des coûts par économie d'échelle, d'envergure et organisationnelles et, d'autre part, d'une anticipation des formes de la concurrence et de la dynamique technologique, permettant un contrôle proactif des relations de la firme avec son environnement (stratégies de coopération). Comme pour le $S B$, les PME $S I$ relèvent en effet d'un modèle de planification ex ante de l'activité (Fernandez, Picory et Rowe, 1993). Dans ce contexte, les contraintes de taux d'intérêt, de structure financière ou de risque bancaire ne paraissent pas constituer de freins sérieux à la dynamique d'investissement de ces firmes ${ }^{20}$. Sur ce point encore, le degré d'intégration bancaire de l'activité paraît largement impliqué.

Pour ces firmes, en effet, la part de l'autofinancement dans le financement de l'investissement est très largement supérieure à la moyenne $(5,82)$ et, corrélativement, les parts du leasing-crédit bail et des prêts bancaires plus réduites (respectivement -3 et $-3,30$ ). Cette structure de financement est liée: 1) au niveau des fonds propres considéré comme suffisant $(9,38)$ et à leur proportion dans les capitaux permanents $(8,98) ; 2$ ) au dynamisme des marchés qui permet de dégager des sources internes de financement de l'investissement. Cela conduit à une autonomie relative par rapport au secteur bancaire dans la mesure où ces PME ne prévoient pas augmenter la part de leur endettement bancaire dans les fonds stables de l'entreprise $(7,68)$. La non-participation de la banque au capital de la firme $(5,54)$, comme l'exclusion de l'éventualité d'une telle participation $(6,08)$, confirment cette recherche d'autonomie, affirmée par ailleurs, au moins dans le choix de la structure de financement $(3,82)$. Conformément à ce qui précède, l'intégration bancaire de l'activité courante est limitée: les PME de cette classe recourent parfois au crédit global d'exploitation $(1,98)$, à l'exclusion de l'escompte $(11,22)$, et privilégient souvent le crédit interentreprises (2). Pourtant, dans la mesure où une part considérable de l'activité de ces firmes est réalisée à l'exportation, elles bénéficient de l'appui financier de la banque $(3,85)$. Au total, la situation financière de ces entreprises paraît relativement autonome parce que contrôlée ex ante, par rapport à l'offre de crédit bancaire; la trésorerie semble saine, puisqu'elle n'a pas fait l'objet de difficultés par le passé $(10,65)$. Enfin, au contraire des $S B$, les négociations des conditions de financement ne portent pas sur les taux $(4,24)$, mais plutôt sur les dates de valeur $(5,70)$ et les commissions $(1,75)$.

L'autonomie de ces firmes n'est cependant que relative. D'une part, parce que les concours bancaires à long ou court terme demeurent très significatifs dans les structures de financement. D'autre part, parce que les relations

20. Les indices de contrainte correspondants sont très largement inférieurs à la moyenne, avec des valeurs tests respectives de $-22,83,-22,31$ et $-24,85$. 
de ces PME avec la banque ne se réduisent pas au financement. Remarquons tout d'abord que le nombre moyen de banques est, pour ces PME, supérieur à la moyenne: 4 à 5 banques $(8,05)$. L'objectif recherché de cette multibancarisation est la complémentarité $(5,12)$. Dans le cadre de l'EDI, les flux d'information transitent dans le sens entreprise-banque $(3,94)$, ce qui correspond, d'une part, à la recherche d'une simplification de la fonction financière $(4,12)$ et, d'autre part, à un souci d'information de la banque dans le cadre d'une relation de confiance $(5,85)$. À cet égard, la transmission d'informations sensibles sur l'entreprise ne semble pas constituer une difficulté $(2,41)$. La contribution la plus importante de la banque à l'activité des $S I$ est en fait à rechercher au niveau des services et conseils: très bon suivi bancaire du risque export $(5,69)$, bonne appréciation de la prestation de la banque en matière de conseils en gestion courante $(3,46)$ et, enfin, très bonne prestation en matière de gestion de trésorerie $(2,27)$.

L'ensemble des caractéristiques qui viennent d'être décrites conduisent alors à d'excellents niveaux des indices de satisfaction, qu'il s'agisse des fonctions bancaires de conseil, de la compréhension des spécificités de l'entreprise, de la gestion du risque ou encore des fonctions bancaires traditionnelles (indices très supérieurs à la moyenne avec des valeurs tests respectives de $8,13 ; 7,56 ; 5,54 ; 4,42)$.

\subsection{Classe des PME SS, dont le degré d'intégration bancaire est très élevé, mais dont l'activité est extrêmement contrainte par le secteur bancaire}

Pour les firmes de cette classe, les caractéristiques sont, d'une part, un niveau légèrement supérieur à la moyenne de l'indice de contrainte de marché $(7,98)$ en liaison avec les niches concurrentielles de spécialité qu'elles parviennent à se ménager en dépit d'une forte exposition à la concurrence et, d'autre part, les scores massivement supérieurs à la moyenne des indices de contraintes de financement: taux d'intérêt $(17,97)$, rentabilité $(17,61)$, risque bancaire $(24,4)$ et structure financière (25). Bien que l'environnement concurrentiel de ces firmes soit relativement certain, elles semblent souffrir tout particulièrement des difficultés conjoncturelles observées depuis 1991, en raison de leur position dans la chaîne de valeur. Ces entreprises sont, en effet, extrêmement spécialisées dans certaines productions et réalisent une grande part de leur activité en sous-traitance.

Ces caractéristiques s'accompagnent d'une structure de financement très particulière. Le niveau des fonds propres est insuffisant $(11,40)$ et la part de l'endettement dans les capitaux permanents est très supérieure à la moyenne $(2,35)$. Les chefs d'entreprises prévoient augmenter cette part $(4,63)$. Les parts 
de l'emprunt bancaire et du leasing-crédit bail dans le financement de l'investissement sont également considérables et très supérieures à la moyenne $(3,73$ et 3,32 ), tandis que celle de l'autofinancement est très inférieure à la moyenne $(-6,51)$. Le financement de l'activité courante s'appuie sur le crédit global d'exploitation $(2,10)$, l'escompte $(11,9)$ et le découvert autorisé $(11,9)$. Cette structure de financements courts et coûteux rend compte, en partie, de nombreux problèmes de trésorerie $(11,36)$ importants $(5,74)$ et très importants $(5,94)$; ce qui suscite une aide conséquente de la banque dans la vérification de la solvabilité de la clientèle de la PME $(2,76)$. Ces éléments permettent de conclure à un degré d'intégration bancaire très élevé de l'activité de ces PME. Ce degré ne correspond cependant pas à un choix. À l'exclusion des taux et modalités de remboursement qui font l'objet de négociation (2,32 et 1,64), les autres conditions (montant des emprunts, commissions, dates de valeurs) ne sont jamais négociées $(1,67 ; 2,95$ et 4,72$)$. Très souvent, la structure de financement est imposée par la banque $(3,83)$ et le financement bancaire s'accompagne de garanties (hypothèques et nantissements) considérées par les PME comme justifiées $(7,53)$. Les garanties sur patrimoine personnel sont cependant dénoncées avec ferveur $(62,4)$. Ces éléments renvoient, nous semble-t-il, à une caractéristique fondamentale des firmes SS (Fernandez, Picory et Rowe, 1993): un modèle de contrôle ex post de l'activité de ces firmes articulé autour du pilotage seul, à partir des «affaires». Compte tenu d'un environnement concurrentiel relativement certain, bien que fluctuant, de l'insertion des firmes dans des niches de spécialité, dans des réseaux de sous-traitance, l'activité ne suscite pas de planification, mais plutôt un ajustement réactif aux formes de la concurrence et à la dynamique technologique, exogène. En période de difficultés conjoncturelles, le retour sur investissement est alors largement compromis ${ }^{21}$, et les problèmes de solvabilité sont plus importants, en partie en raison de l'inexistence d'une planification ex ante de l'activité.

Dans le cadre de ce contexte, les relations plus qualitatives avec les banques sont conflictuelles par rapport aux PME des classes précédentes (désaccords sérieux : 2,52 ) de telle sorte que le degré de confiance mutuelle est faible $(2,08)$ ou très faible $(3,37)$. L'une des raisons fondamentales de ces conflits est l'asymétrie d'information: l'EDI bancaire n'est pas pratiquée $(3,93)$ et les PME de cette classe n'échangent pas d'information sensible avec leurs banques $(5,53)$. La multibancarisation de ces PME est plus rare: le nombre de banques est en effet très sensiblement inférieur à la moyenne $(-6,52)$. La multibancarisation est recherchée afin d'assurer la continuité des financements $(3,78)$. Dans le cadre des relations avec la banque principale, ou l'unique

21. La rentabilité de ces PME par rapport à la moyenne du secteur est faible $(6,18)$ ou moyenne $(4,37)$. 
banque selon le cas, le professionnel chargé du suivi de la PME est plutôt un chargé de clientèle entreprise $(3,59)$. À l'exclusion de la gestion de la trésorerie où la performance de ce professionnel est bien appréciée $(4,48)$, les compétences de ce dernier sont très mal évaluées en matière de financement de l'investissement $(3,43)$, de placements $(4,08)$, d'information et de conseils sur la gestion courante $(7,35)$, de stratégies à long terme de l'entreprise $(3,78)$, de gestion des risques clients $(5,34)$ ou d'exportation $(4,26)$. Globalement, la compréhension des spécificités de l'entreprise semble très mauvaise $(11,1)$. Les indicateurs synthétiques de satisfaction et de confiance sont dès lors tous très inférieurs à la moyenne.

\subsection{Synthèse}

À l'exclusion des firmes $S T$ que nous n'avons pu singulariser avec suffisamment de fiabilité, les distinctions qui viennent d'être introduites en matière de degré d'intégration bancaire de l'activité des PME permettent d'étayer l'argumentation développée au début de la section.

Le tableau 3 tend en effet à accréditer l'idée d'une sélectivité, au moins implicite, de l'offre de crédit bancaire au tissu productif. Moins l'organisation industrielle paraît présenter un risque de financement élevé, plus l'offre bancaire est élastique et semble s'adapter aux tensions marchandes qui affectent les entreprises et singulièrement les PME. Le comportement du système bancaire reste bien guidé par le triptyque risque-rentabilité-solvabilité. Toutefois, et de manière générale, lorsque le système bancaire perçoit un risque supérieur et de gravité croissante, il paraît exercer une contrainte croissante sur son encours de crédits. De ce fait, la structure de la dette tend à se déformer progressivement au profit des concours bancaires courts, tandis que les niveaux de garanties bancaires sur l'appareil productif et le patrimoine personnel des chefs d'entreprises tendent à devenir de plus en plus élevés, et que les taux d'intérêts tendent, eux-mêmes, à atteindre le seuil butoir fixé par la législation en matière de taux d'usure.

Si cette interprétation est correcte, le système bancaire tendrait alors à privilégier une évaluation catégorielle du risque de financement au détriment d'une approche individuelle du risque de défaillance. Le principal critère d'accès au crédit bancaire, comme la différenciation du coût des concours, serait alors adossé au montant des crédits ainsi qu'à celui des encours, en fonction de seuils d'endettement, variables selon les organisations industrielles dans lesquelles s'inscrivent les firmes, c'est-à-dire selon le poids de l'aléa, statistiquement observable qui intervient entre anticipation (ex ante) et validation marchande (ex post). Cet adossement n'est probablement pas explicité dans les approches classiques et actuarielles du risque de financement. Il serait en 
TABLEAU 3

Risque de défaillance et degré d'intégration bancaire

\begin{tabular}{ccc}
\hline Organisation & $\begin{array}{c}\text { Taux de } \\
\text { défaillance } \\
\text { industrielle }\end{array}$ & $\begin{array}{c}\text { Degré d'intégration bancaire de l'activité } \\
\text { (Investissement et exploitation) } \\
\text { (Industrie 1993) }\end{array}$ \\
(sur un échantillon d'entreprises 1993)
\end{tabular}

$S B$

$S I$
Très élevé à court et long terme. Firmes faiblement contraintes par le système bancaire.

Niveau élevé de crédits longs et de fonds propres.

Part relativement limitée du leasing-crédit bail et de l'autofinancement dans le financement des investissements. Niveau élevé de crédits courts : crédit global d'exploitation, crédit en devises, crédit interentreprises avec mobilisation de créances (escompte).

Difficultés de trésorerie éventuelles résolues avec l'aide de la banque.

Structure de financement choisie. Négociation sur les seuls taux d'intérêt.

Très faible multibancarisation, voire unicité de la banque.

Faibles asymétries d'information.

Forte satisfaction et degré élevé de confiance mutuelle.

Élevé à court et long terme. Firmes faiblement contraintes par le système bancaire. Recherche d'autonomie des financements.

Niveau élevé de crédits longs et des fonds propres.

Part relativement limitée du leasing-crédit bail

dans le financement des investissements. Privilège accordé à l'autofinancement.

Niveau élevé de crédits courts : crédit global d'exploitation, crédit en devises, crédit interentreprises sans mobilisation de créances (escompte).

Difficultés éventuelles de trésorerie résolues en interne.

Structure de financement choisie. Négociation sur les seuls taux d'intérêt.

Très forte multibancarisation de complémentarité.

Faibles asymétries d'information.

Forte satisfaction et degré élevé de confiance mutuelle.

Très élevé à court et long terme, structure déformée sur le court terme et firmes fortement contraintes par le système bancaire.

Niveau très élevé de crédits longs et insuffisance des fonds propres.

Part du leasing-crédit bail très élevée. Part limitée de l'autofinancement dans le financement des investissements.

Niveau élevé de crédits courts : crédit global d'exploitation, découvert autorisé, crédit interentreprises avec mobilisation de créances (escompte).

Nombreuses difficultés de trésorerie.

Structure de financement imposée par la banque.

Négociation sur les seuls taux d'intérêt et plan

d'amortissement des prêts.

Fortes garanties bancaires sur l'appareil de production et biens personnels.

Multibancarisation plus rare et pour assurer la continuité des financements.

Fortes asymétries d'information.

Insatisfaction et degré très faible de confiance mutuelle.

Données insuffisantes pour une caractérisation fiable.

Source : C. Picory. 
fait véhiculé par l'intermédiaire des demandes de crédits bancaires de trésorerie. La différence entre les valeurs escomptées et les grandeurs réalisées (c'est-à-dire le risque industriel) est imputable soit aux fluctuations conjoncturelles, soit à une incertitude radicale structurelle sur la dynamique des marchés et de la technologie, soit encore à des prévisions erronées. Cette différence se traduit financièrement par une augmentation des besoins en fond de roulement et une dégradation de la trésorerie, si les conséquences financières de l'écart observé ne sont pas répercutées sur les prix de vente.

Or, les pratiques bancaires paraissent telles que le risque de financement (le risque de défaillance) est considéré comme s'accroissant avec les demandes supplémentaires de crédits de trésorerie. Le risque structurel lié à l'organisation industrielle dans laquelle s'inscrit l'activité de la firme induirait une structure de financement déformée sur le court terme et serait alors traité par le système bancaire de manière purement routinière par une limitation de l'encours des crédits, une différenciation des taux d'intérêts et l'imposition de garanties importantes.

\section{Conclusion}

Nous avons circonscrit l'objet de cet article au test de l'hypothèse suivante:

$\boldsymbol{H}$ : Il existerait une relation entre la nature de l'organisation industrielle, définie en fonction de configurations particulières de caractéristiques de l'activité et à laquelle appartiennent la PME, la nature, l'ampleur et l'intensité de l'intégration bancaire de l'activité productive et les divers niveaux de risque (de financement)-rentabilité-solvabilité.

L'ensemble des développements précédents nous permet alors de conclure.

La section 3 montre que le critère de l'organisation industrielle constitue un critère pertinent de différenciation du risque de financement. Ce dernier tend principalement à varier en raison inverse par rapport à l'intensité et aux performances des processus innovants et, dans une moindre mesure, en raison directe avec le degré d'exposition à la concurrence.

La section 4 montre que dans le contexte d'un modèle de «banque-acte», les concours du système bancaire au système productif restent adossés au triptyque risque-rentabilité-solvabilité. Sous cette contrainte, à chaque catégorie d'organisation industrielle, c'est-à-dire à chaque «classe» de risque structurel, correspond une structure de financement particulière. Dans le cadre d'une approche tendant à substituer une analyse catégorielle du risque de financement, ainsi qu'une sélection des encours par montants, à une évaluation 
individuelle du risque, la différenciation en termes d'encours et de coût des concours s'accompagne d'une déformation de la structure de la dette des entreprises, et singulièrement des PME, au profit des concours les plus courts et les plus coûteux, à mesure que le risque catégoriel de défaillance croît et que les demandes de crédits de trésorerie signalent un risque industriel (différence entre grandeurs prévues et grandeurs réalisées) croissant.

Les relations qui viennent d'être établies montrent qu'à chaque catégorie d'organisation industrielle peut être associé un niveau de risque industriel auquel correspond un niveau de risque de financement (défaillance) ainsi qu'un degré spécifique d'intégration bancaire de l'activité productive. L'hypothèse $\boldsymbol{H}$ ne saurait, par conséquent, être rejetée.

Cette étude tend à montrer que les relations entre PME (peu importe la taille ?) et secteur bancaire sont sous-tendues, en premier lieu, par la nature différenciée de l'organisation industrielle dans laquelle s'inscrit la firme et, en second lieu, par l'aléa structurel et statistiquement observable qui intervient dans la définition du risque industriel associé à chaque catégorie. Afin d'affiner l'analyse, il conviendrait cependant:

- de caractériser plus précisément, à l'échelle de l'industrie et selon une approche plus dynamique, la structure financière des entreprises appartenant à chaque catégorie d'organisation industrielle, en particulier, par rapport aux caractéristiques relevées par Bardos (1990), Blazy, Charlety et Combier (1993): dégradation de la rentabilité des entreprises défaillantes, déséquilibre de leur structure de financement, faiblesse de leur taux d'investissement et allongement symptomatique de leurs délais fournisseurs;

- d'établir avec plus de précision encore l'articulation entre risque industriel et risque de financement, c'est-à-dire la manière dont l'horizon et le degré de réalisation des prévisions influent sur les structures et les comportements d'endettement des PME.

Autant de questions pour un agenda de recherche stimulant... 


\section{Annexe}

À partir des réponses à un questionnaire, des indices ont été construits afin de quantifier l'appréciation de l'entrepreneur sur: 1) les contraintes pesant sur son effort d'investissement; 2) la satisfaction quant à la qualité des prestations offertes par la banque principale; et 3 ) la confiance que la PME manifeste à l'égard de sa banque principale. Pour ce faire, chaque question recueillant l'appréciation de la PME sur ses contraintes ou sur la qualité des prestations, ou sur la confiance est réévaluée selon un score, normalisé à 1 pour faciliter les comparaisons entre deux indices. Les indices croissent avec l'intensité des contraintes, de la satisfaction et de la confiance.

L'indice de contrainte pesant sur l'investissement regroupe les contraintes macroéconomique (contrainte de taux d'intérêt), mésoéconomique (contraintes de dynamique de marché: marché stagnant/fluctuant et concurrence trop forte), microéconomique (contrainte de rentabilité) et bancaire (structure financière: impact du manque de fonds propres et de l'endettement excessif sur l'investissement; risque bancaire: impact des conditions de financement bancaire et des garanties exigées par la banque sur l'investissement). Un indicateur évalue la contrainte globale pesant sur la PME. Il est construit afin de privilégier les contraintes d'ordre bancaire et de minimiser le rôle de la contrainte macroéconomique. Sa valeur oscille entre 0 et 10 .

L'indice de satisfaction de la PME évalue les prestations offertes par la banque par rapport à la fonction bancaire traditionnelle (gestion de la trésorerie, du financement des investissements, de placements), à la fonction bancaire de conseil (conseil en gestion courante et en stratégie à long terme), à la fonction bancaire de gestion du risque (gestion du risque client et du risque export), à la fonction de compréhension de la spécificité de la firme, enfin. Un indicateur évalue la satisfaction globale de la PME à l'égard des prestations bancaires. Il est construit de façon à minimiser l'importance des prestations bancaires les plus courantes.

L'indicateur de confiance de la PME à l'égard de sa banque principale est construit en tenant compte de l'appréciation de la probabilité de l'entrée d'une banque dans le capital (de très envisageable à exclu), de l'évaluation du degré de confiance mutuelle PME-banque principale (de très fort à très faible) et de l'échange d'informations sensibles de la PME vers la banque (de très facilement à très rarement). L'indicateur de confiance est pondéré par un demi si la PME a été en profond désaccord avec la banque dans le passé, et par 1 si aucun désaccord n'est intervenu, cela afin de minimiser l'impact des réponses dictées par la rancœur. 


\section{Bibliographie}

BARDos, M. (1990), «Le crédit plus cher pour les petites entreprises», Économie et Statistiques, n*236, p. 51-64.

Blazy, R., P. Charlety et J. Combier (1993), «Les défaillances d'entreprises: des difficultés visibles plusieurs années à l'avance», Economie et Statistique, $\mathrm{n}^{\text {os }}$ 268-269, p. 101-111.

CARTElier, J. (1991), « Monnaie et système de paiement: le problème de la formation de l'équilibre", Revue Française d'Économie, vol. 4, n 3, p. 3-37.

COASE, R.H. (1937), «The nature of the firm», Economica, no 4, p. 386-405.

COASE, R.H. (1972), «Industrial organization: a proposal for research », dans V.R. Fuchs (éd.) (1972), Policy Issues and Research Opportunities in Industrial Organization, NBER General Series, $\mathrm{n}^{\circ}$ 96, Traduction française dans Revue d'Économie Industrielle, $\mathrm{n}^{\circ}$ 58, p. 15-27.

Combier, J. (1994), «Les défaillances en 1993 », INSEE Première, n 335, p. 1-4.

Dosi, G., K. PAvitT et L. SoETE (1985), The Economics of Innovation and International Trade, Brighton, Wheatsheaf.

FERnANDEZ, V., C. PICORY et F. Rowe (1993), «Outils de gestion et espaces concurrentiels des PME», Congrès International Francophone sur la PME, Carthage, 28-29 octobre, à paraître dans Revue Internationale PME, 1996, vol. 9, nº 1 .

KNight, F. (1921), Risk, Uncertainty and Profit, Boston, Houghton Mifflin.

MARICIC, A. et C. PICORY (1991), «La place du système financier dans les analyses d'économie industrielle», dans R. Arena, L. Benzoni, J. de Bandt et P.M. Romani (éd.) (1991), Traité d'Économie Industrielle, $2^{c}$ éd., Paris, Economica.

PAVITT, K. (1984), «Sectoral patterns of technical change: towards a taxonomy and a theory», Research Policy, n 13, p. 343-373.

PICORY, C. (1990a ), Prix et environnement concurrentiel des PME, Paris, ENST.

PICORY, C. (1990b), «PME, comportements d'innovation et espaces concurrentiels », Communication au colloque La PME: objet de recherche pertinent?, TETRAUniversité de Lyon II, 30-31 mai.

PICORY, C. (1994), «PME, incertitude et organisation industrielle: une mise en perspective théorique», Revue d'Économie Industrielle, $\mathrm{n}^{0}$ 67, p. 40-58.

PICORY, C. et B. GEFFROY (1994), «Organisation industrielle et degré d'intégration bancaire de l'activité des PME», Actes du colloque Banks and Growth Financing, Lisbonne, 26-27 avril.

PICORY, C. et B. GefFroy (1995), «Degré d'intégration bancaire des PME: une approche par l'organisation industrielle», Revue Économique, n” 2, p. 365-392.

RIVAUT-DANSET, D. (1991), «La relation banque-entreprise: une approche comparée», Revue d'Économie Financière, n"16, p 105-118. 